Pure and Applied Mathematics Quarterly

Volume 9, Number 3

$493-506,2013$

\title{
On the Asymptotic Stability of Stationary Lines in the Curve Shortening Problem
}

\author{
Xiao-Liu Wang and Wei-Feng Wo*
}

\begin{abstract}
The asymptotic stability of the stationary lines in the curve shortening problem was studied in Nara-Taniguchi [NT2] when the initial curve is a graph over the stationary line. The result is extended to a class of nongraphic initial curves.

Keywords: curve shortening problem, curvature flow, asymptotic behavior, foliation, Sturm oscillation theorem.
\end{abstract}

\section{INTRODUCTION}

In the curve shortening problem (CSP) a family of plane curves $\gamma_{t}$ evolves under the equation

$$
\frac{\partial \gamma_{t}}{\partial t}=k \nu
$$

where the unit normals $\nu$ and the curvature $k$ are defined by $\ddot{\gamma}=k \nu$ as usual in $\mathbb{R}^{2}$ and $\ddot{\gamma}$ denotes the second order derivative of $\gamma$ with respect to arclength. As is well-known, aside from being the simplest case of curvature flows, the (CSP) also arises in applied areas such as phase transitions, Visintin $[\mathrm{V}]$ and image processing, Sapiro [S]. The (CSP) starting with a closed, embedded curve was studied by Gage-Hamilton [GH] who showed that it shrinks to a point in finite

Received September 27, 2010.

2000 Mathematics Subject Classification Primary 53C44, Secondary 35B05, 35B35, 35B40, $53 \mathrm{C} 12$.

This work was partially supported by the National Natural Science Foundation of China 11101078, 11171064, 11001049 and the Natural Science Foundation of Jiangsu Province BK2011583.

*Corresponding author 
time, which, after blowing up, becomes a circle. To complete the study, Grayson [G] showed that any closed, embedded curve flows into a convex one in finite time under the (CSP). Thus the problem for closed, embedded curves is completely understood. For complete, non-compact embedded curves the (CSP) was studied subsequently in several works. In Ecker-Huisken [EH1],[EH2] it was shown that when the initial curve is an entire graph, this problem has a global solution which remains as graphs for all time. (In fact, in these works the higher dimensional version of the (CSP), the mean curvature flow, is studied.) They further established an asymptotic result asserting that the curve evolves to an expanding self-similar solution when the initial graph satisfies some growth conditions. See Stavrou [St] and Ishimura [I] for related results.

A straight line, usually taken to be the $\mathrm{x}$-axis, is a stationary solution of the (CSP). The convergence to straight lines was studied by several authors. In Polden $[\mathrm{P}]$ ( see also Huisken $[\mathrm{H}]$ ) it is shown that any embedded curve whose ends are $C^{1}$-asymptotic to the $x$-axis converges to the $x$-axis smoothly as $t \rightarrow \infty$. In Nara-Taniguchi [NT1], assuming $\gamma_{0}$ is an entire graph $\left(x, u_{0}(x)\right)$, it is established that $u(x, t)$, where $\gamma_{t}=(x, u(x, t))$, converges uniformly to zero provided $u_{0} \in C^{2, \alpha}(\mathbb{R})$ for some $\alpha \in(0,1)$ and $u_{0}$ tends to zero uniformly at the ends. Under the additional assumption that $u_{0} \in L^{1}(\mathbb{R})$, they also obtained the decay estimate

$$
\sup _{x}|u(x, t)| \leq C(1+t)^{-1 / 2}, t>0 .
$$

In all of these works, the convergence to straight lines or self-similar solutions had been established under certain uniform growth or decay conditions of the initial curve at the ends. Thus it is surprising that Nara-Taniguchi [NT2] proved the following result: Assuming that the initial graph $u_{0} \in C^{2, \alpha}(\mathbb{R})$ (for some $\alpha \in(0,1))$ and satisfies

$$
\lim _{R \rightarrow \infty} \sup _{x_{0}} \frac{1}{2 R}\left|\int_{x_{0}-R}^{x_{0}+R} u_{0}(x) d x\right|=0,
$$

they showed that $u(x, t)$ converges to zero uniformly. Moreover, the condition (1) is also necessary for an initial $C^{2, \alpha}$-function $u_{0}$ whose solution $u(t)$ of the (CSP) decays to 0 uniformly. 
In this paper we extend this result to embrace certain classes of complete, non-compact embedded curves. To formulate our result we first express (1) in a form which is valid for non-graphic curves. Let $\gamma(p)=(x(p), y(p))$ be a smooth, properly embedded curve contained in $\mathbb{R} \times(-M, M)$ for some positive $M$. As it is properly embedded, we can choose a parametrization so that $\gamma(p) \rightarrow \pm \infty$ as $p \rightarrow \pm \infty$. Without loss of generality we may also assume $x(0)=0$. For any given number $l>0$, divide $\mathbb{R}$ into intervals, $I_{n}=[(2 n-1) l,(2 n+1) l], n \in \mathbb{Z}$. Starting from $\gamma(0)$, as $p$ increases the curve $\gamma(p)$ will eventually leave $I_{0}$ by entering $I_{1}$ at $p_{1}$. Similarly, we define $p_{-1}$ the last time the curve touches $x=-l$ and enters $I_{-1}$. We denote $\gamma^{0}=\left.\gamma\right|_{\left[p_{-1}, p_{1}\right]}$. Similarly, we can define $\gamma^{n}=\left.\gamma\right|_{\left[p_{2 n-1}, p_{2 n+1}\right]}$. For each $\gamma^{n}$ we extend it to be a closed curve $c^{n}$ by adjoining to it the line segments in $x=(2 n \pm 1) l$ and the $x$-axis (See Figure 1 ).

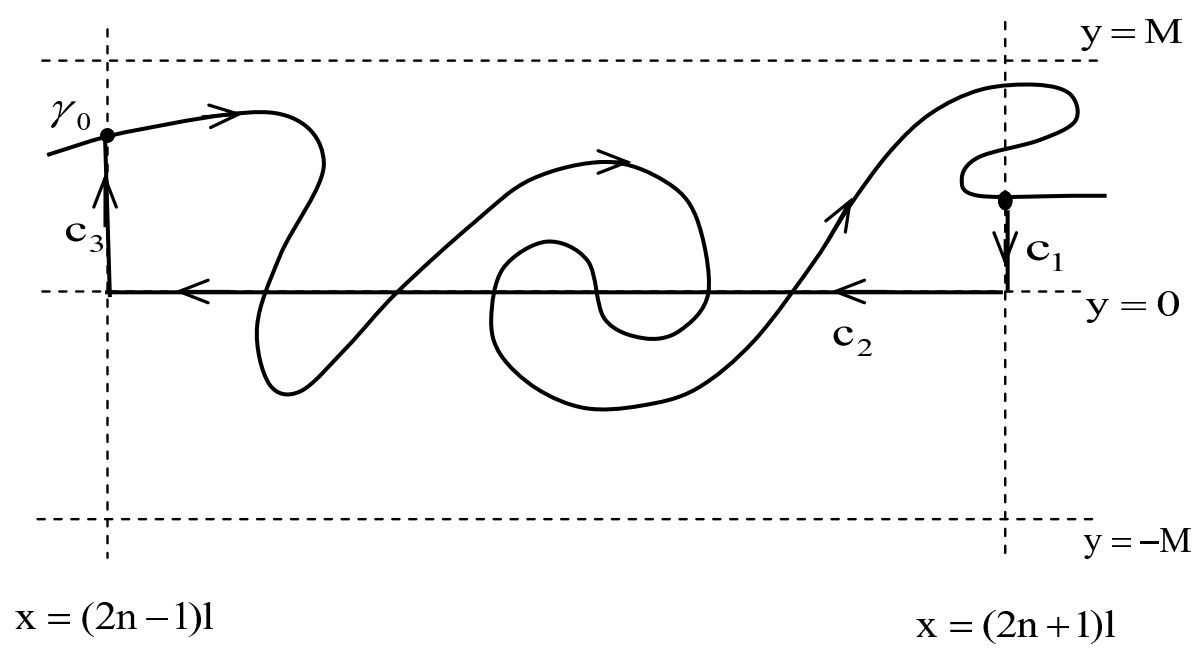

Figure 1. The closed curve $c^{n}$.

We define the "signed area" of $\gamma$ over $I_{n}, A_{n}(\gamma)$, to be

$$
A_{n}(\gamma)=\frac{1}{2} \int_{c^{n}}(y d x-x d y)
$$

Note that when $\gamma^{n}=(x, u(x))$ is a graph over $[(2 n-1) l,(2 n+1) l]$,

$$
A_{n}(\gamma)=\int_{(2 n-1) l}^{(2 n+1) l} u(x) d x
$$


For each $N \in \mathbb{Z}$, denote $A_{N, n}(\gamma)=\sum_{j=N-n}^{N+n} A_{j}(\gamma)$ the signed area of $\gamma$ over $[(2 N-2 n-1) l,(2 N+2 n+1) l]$. We shall replace $(1)$ by the following condition

$$
\lim _{n \rightarrow \infty} \sup _{N} \frac{1}{(4 n+2) l}\left|A_{N, n}\left(\gamma_{0}\right)\right|=0 .
$$

When $\gamma_{0}=\left(x, u_{0}(x)\right)$ is confined to a strip and $u_{0}$ is uniformly continuous on the real line, it is clear that (1) holds if and only if (2) holds (for any fixed $l$ ). Now taking $M=1$ for simplicity, we state

Theorem 1. Suppose $\mathcal{C}_{1}, \mathcal{C}_{2}, \cdots, \mathcal{C}_{l}\left(l \in \mathbb{N}^{*}\right)$ are given embedded $C^{2, \alpha}$-curves, which are bounded and contained in $\mathbb{R} \times(-1,1)$ for some $\alpha \in(0,1)$. Let $\gamma_{0}$ be a properly embedded $C^{2, \alpha}$-curve contained in $\mathbb{R} \times(-1,1)$. Assume further that there exist a sequence of disjoint intervals $\left\{\left(a_{n}, b_{n}\right)\right\}_{n=1}^{N}(N<\infty$ or $=\infty)$, and a small number $\varepsilon_{0}>0$ such that

$\gamma_{0}$ has exactly the same shape with one of $\mathcal{C}_{i}(1 \leq i \leq l)$ over every interval $\left(a_{n}, b_{n}\right), \forall 1 \leq n \leq N$,

and

$\gamma_{0}$ is graphic over the set $\mathbb{R} \backslash\left(\cup_{n=1}^{\infty}\left[a_{n}+\varepsilon_{0}, b_{n}-\varepsilon_{0}\right]\right)$.

Let $\gamma_{t}$ be the global solution of the (CSP) starting at $\gamma_{0}$. Then if (2) holds, there is a time $T>0$ such that $\gamma_{t}$ evolves into an entire graph $\left(x, u^{*}(x)\right)$ at time $T$ and (1) holds for $u_{0}=u^{*}$. Consequently, $\gamma_{t}$ converges to $x$-axis uniformly as $t \rightarrow \infty$.

The existence and uniqueness of a global solution to the (CSP) for smooth initial curves satisfying the assumptions in the theorem is established in Chou-Zhu [CZ1]. Our proof of this theorem consists of two parts. First, we show that the condition (2) is preserved under the (CSP). Second, we show that $\gamma_{t}$ will evolve into an entire graph in finite time. After showing these, the convergence result follows from Nara-Taniguchi [NT2].

To conclude the introduction we point out some works on the asymptotic stability of other invariant solutions of the (CSP). One may consult Altschuler-Wu [AW] for traveling waves and Hungerbühler-Smoczyk [HS] for spiral waves.

The author would like to thank Prof K.-S. Chou for his suggestion and encouragement during the preparation of this work. We are grateful to a referee whose comments have helped improve the presentation of this paper. 


\section{Area Evolution}

Let $\gamma_{0}$ be a smooth, embedded curve confined to $\mathbb{R} \times(-1,1)$, which are graphs over some open intervals containing $R_{1}$ and $R_{2}$ respectively with $R_{1}<R_{2}$. By the Sturm oscillation theorem (see, e.g. [A1], or chapter 1 in Chou-Zhu [CZ2]), $\gamma_{t}$ remains as graphs over the same intervals. Moreover, the vertical lines $x=R_{1}, R_{2}$ are transversal to the graph of $\gamma_{t}$ for all time.

Denote by the same notation $\gamma_{0}$ the portion of $\gamma_{0}$ inside $\left[R_{1}, R_{2}\right] \times(-1,1)$. Then $\gamma_{0}$ is regarded as a curve starting at a point on $x=R_{1}$ and ending up at a point on $x=R_{2}$. With this orientation, together with the $x$-axis and the two vertical lines $x=R_{1}, R_{2}$, we get a closed curve $c$. See Figure 1 for a similar situation. Here $\gamma_{0}$ is completely contained inside $\left[R_{1}, R_{2}\right] \times(-1,1)$. We denote the restriction of $\gamma_{t}$ in this region by the same notation. Then the curve $\gamma_{t}(p)=(x(p, t), y(p, t))$ intersects $x=R_{1}, R_{2}$ respectively at $p=p_{*}(t)$ and $p=p^{*}(t)$. We have

$$
\begin{aligned}
& x\left(p_{*}(t), t\right)=R_{1}, \\
& x\left(p^{*}(t), t\right)=R_{2} .
\end{aligned}
$$

By transversality, $p_{*}$ and $p^{*}$ are differentiable and

$$
x_{p} \frac{d p_{*}}{d t}+x_{t}=0, \quad x_{p} \frac{d p^{*}}{d t}+x_{t}=0,
$$

respectively at the intersection points. We use the parametrization $p \in\left[p_{*}(t), p^{*}(t)\right]$ to describe $\gamma_{t}$. For the other boundaries, we use the following parameterizations,

$$
\begin{aligned}
& c_{1}(y): y \text { runs from } y\left(p^{*}(t), t\right) \text { to } 0 \\
& c_{2}(x): x \text { runs from } R_{2} \text { to } R_{1} ; \\
& c_{3}(y): y \text { runs from } 0 \text { to } y\left(p_{*}(t), t\right) .
\end{aligned}
$$

It follows that

$$
\begin{aligned}
2 A(t) & =\int_{c}(y d x-x d y) \\
& =\left(\int_{\gamma_{t}}+\int_{c_{1}}+\int_{c_{2}}+\int_{c_{3}}\right)(y d x-x d y) \\
& =\int_{p_{*}(t)}^{p^{*}(t)}\left(y x_{p}-x y_{p}\right) d p+R_{2} y\left(p^{*}(t), t\right)-R_{1} y\left(p_{*}(t), t\right),
\end{aligned}
$$


where $A(t)$ is the signed area of $\gamma_{t}$ over $\left[R_{1}, R_{2}\right]$. We would like to compute $d A / d t$ at any $t_{0} \geq 0$. To simplify the computations we assume $\gamma_{t_{0}}$ is parameterized by the arc length. We have

$$
\begin{aligned}
& \left.\frac{d}{d t} \int_{p_{*}(t)}^{p^{*}(t)}\left(y x_{p}-x y_{p}\right) d p\right|_{t=t_{0}} \\
= & \int_{p_{*}\left(t_{0}\right)}^{p^{*}\left(t_{0}\right)}\left\langle\left(x_{t}, y_{t}\right),\left(-y_{p}, x_{p}\right)\right\rangle+\left\langle(x, y),\left(-y_{p t}, x_{p t}\right\rangle d p+\right. \\
& \left(y x_{p}-x y_{p}\right)\left(p^{*}\left(t_{0}\right), t_{0}\right) p_{t}^{*}\left(t_{0}\right)-\left(y x_{p}-x y_{p}\right)\left(p_{*}\left(t_{0}\right), t_{0}\right) p_{* t}\left(t_{0}\right) \\
= & 2 \int_{p_{*}\left(t_{0}\right)}^{p^{*}\left(t_{0}\right)} k d p-\left.k\left(x x_{p}+y y_{p}\right)\right|_{\left(p_{*}\left(t_{0}\right), t_{0}\right)} ^{\left(p^{*}\left(t_{0}\right), t_{0}\right)}+ \\
= & 2 \int_{p_{*}\left(t_{0}\right)}^{p^{*}\left(t_{0}\right)} k d p-R_{2} \frac{k\left(p^{*}\left(t_{0}\right), t_{0}\right)}{x_{p}\left(p^{*}\left(t_{0}\right), t_{0}\right)}+R_{1} \frac{k\left(p_{*}\left(t_{0}\right), t_{0}\right)}{x_{p}\left(p_{*}\left(t_{0}\right), t_{0}\right)}
\end{aligned}
$$

after using $x_{t}=-k y_{p}, y_{t}=k x_{p}$ and (2.1) at $t=t_{0}$. On the other hand,

$$
\begin{aligned}
& \left.\frac{d}{d t}\left(R_{2} y\left(p^{*}(t), t\right)-R_{1} y\left(p_{*}(t), t\right)\right)\right|_{t=t_{0}} \\
= & \left.R_{2}\left(y_{p} p_{t}^{*}+y_{t}\right)\right|_{\left(p^{*}\left(t_{0}\right), t_{0}\right)}-\left.R_{1}\left(y_{p} p_{* t}+y_{t}\right)\right|_{\left(p_{*}\left(t_{0}\right), t_{0}\right)} \\
= & \left(\frac{R_{2} k\left(p^{*}\left(t_{0}\right), t_{0}\right)}{x_{p}\left(p^{*}\left(t_{0}\right), t_{0}\right)}-\frac{R_{1} k\left(p_{*}\left(t_{0}\right), t_{0}\right)}{x_{p}\left(p_{*}\left(t_{0}\right), t_{0}\right)}\right)
\end{aligned}
$$

Therefore,

$$
\frac{d}{d t} A(t)=\int_{p_{*}(t)}^{p^{*}(t)} k d p .
$$

The integral on the right of this formula is simply the difference in the tangent angles of $\gamma_{t}$ at the intersection points at $x=R_{2}$ and $R_{1}$. It follows that

$$
\left|\frac{d}{d t} A\left(t_{0}\right)\right| \leq \pi
$$

Since $t_{0}$ is arbitrary, for any fixed $t_{1} \geq 0$, it holds that

$$
\left|A\left(t_{1}\right)\right| \leq|A(0)|+\pi t_{1} .
$$


We now show that (2) is preserved by the evolution. Consider a division of $\mathbb{R}$ into intervals of the form, $[(2 n-1) R,(2 n+1) R)], n \in \mathbb{Z}$. For $\varepsilon>0$, there exists a large $n_{0}$, such that for all $N$,

$$
\frac{1}{2 n}\left|A_{N, n}\left(\gamma_{0}\right)\right|<\frac{\varepsilon}{2}, \forall n \geq n_{0} .
$$

Taking $R_{1}=(2 N-2 n-1) R$ and $R_{2}=(2 N+2 n+1) R$, we apply $(2.2)$ to $A\left(t_{1}\right)$ $=A_{N, n}\left(\gamma_{t_{1}}\right)$ to obtain

$$
\frac{1}{2 n}\left|A_{N, n}\left(\gamma_{t_{1}}\right)\right|<\frac{1}{2 n}\left(\left|A_{N, n}\left(\gamma_{0}\right)\right|+\pi t_{1}\right)<\varepsilon,
$$

if we further take $n_{0} \geq \pi t_{1} / \varepsilon$. We conclude that (2) continues to hold at $\gamma_{t_{1}}$ for any $t_{1}>0$.

Note that (2.2) still holds without the transversality condition at $R_{1}$ and $R_{2}$. Indeed, in the proof, if the vertical lines are not transversal at $R_{1}$ and $R_{2}$, we may find $\left\{\varepsilon_{j}\right\}_{j=1}^{\infty}$ with $\varepsilon_{j} \rightarrow 0$ such that $x=R_{1}+\varepsilon_{j}, R_{2}+\varepsilon_{j}$ are transversal to the initial curve by Sard's theorem. As above we have

$$
\left|\tilde{A}\left(t_{1}\right)\right| \leq|\tilde{A}(0)|+\pi t_{1}
$$

where $\tilde{A}\left(t_{1}\right)$ is the area enclosed by the $x$-axis, the two vertical lines $x=R_{1}+$ $\varepsilon_{j}, R_{2}+\varepsilon_{j}$ and $\gamma_{t_{1}}$. Then it's easy to observe that

$$
\left|A\left(t_{1}\right)\right| \leq|A(0)|+\pi t_{1}+8 \varepsilon_{j},
$$

and hence (2.2) continues to hold after letting $\varepsilon_{j} \rightarrow 0$.

\section{Evolving into an Entire Graph}

We shall use the method of foliations to show that the (CSP) for any initial curve satisfying the assumption of the theorem evolves into an entire graph in finite time.

For convenience, we may assume that $\left(a_{1}, b_{1}\right)=(-R, R)$ with $R>2$ and $\varepsilon_{0}=1$. Then $\gamma_{t}$ is a graph on the intervals $(-R-1,-R+1)$ and $(R-1, R+1)$. We focus on the behaviour of the solution on a typical cell $(-R, R) \times(-1,1)$. Our aim is to construct a foliation $\mathcal{F}$ of $\left[-R-\frac{1}{2}, R+\frac{1}{2}\right] \times[-2,2]$ consisting of "vertical leaves" $\Gamma_{\mu}, \mu \in\left[\mu_{1}, \mu_{2}\right]$, satisfying 
(1) For each $\mu$, the curve $\Gamma_{\mu}(y): y \in[-1,1]$ is smooth, and $\mu \mapsto \Gamma_{\mu}$ is continuous from $\left[\mu_{1}, \mu_{2}\right]$ to $C^{2, \alpha}([-1,1])$,

(2) All $\Gamma_{\mu}$ are mutually disjoint and cover the set $\left[-R-\frac{1}{2}, R+\frac{1}{2}\right] \times[-2,2]$,

(3) $\Gamma_{\mu}^{1}(1)=\Gamma_{\mu}^{1}(-1)$ where $\Gamma_{\mu}=\left(\Gamma_{\mu}^{1}, \Gamma_{\mu}^{2}\right)$ for all $\mu$, and

(4) each $\Gamma_{\mu}$ intersects $\gamma_{0}$ transversally.

It is evident that such foliation exists. However, in the following we sketch a construction in which the dependence of regularity of the foliation on the initial curve is carefully displayed. And the constructed foliations guarantee that $\gamma_{t}$ evolves into a graph over $[-R, R]$ at a time $T$ depending (but not only) on the $C^{2, \alpha}$-regularity of $\gamma_{0}$ in the cell $(-R, R) \times(-1,1)$.

Let $D=(-R-1, R+1) \times\left(-\frac{3}{2}, 2\right)$ and denote the restriction of $\gamma_{0}$ on $D$ still by $\gamma_{0}$. The curve $\gamma_{0}$ divides $D$ into two regions $D_{+}$and $D_{-}$with $D_{+}$lying on the left of $\gamma_{0}$ and $D_{-}$on its right. Denote the four corners of $D_{+}$by $v_{1}=\gamma_{0} \cap\{x=$ $-R-1\}, v_{2}=\gamma_{0} \cap\{x=R+1\}, v_{3}=(R+1,2)$ and $v_{4}=(-R-1,2)$. The following lemma displays the dependence of regularity of the foliation on the initial curve. It guarantees that a $C^{2, \alpha}$ curve will yield a $C^{2, \alpha}$ foliation.

Lemma 3.1. There is a conformal diffeomorphism $\Phi_{+}$mapping $D_{+}$onto the cube $(-1,1) \times(0, q)$ where $q>0$ so that the corners $v_{1}, v_{2}, v_{3}$ and $v_{4}$ are mapped to $(-1,0),(1,0),(1, q)$ and $(-1, q)$ respectively. $\Phi_{+}$extends as a conformal diffeomorphism on $\overline{D_{+}}$away from the corners. Moreover, the $C^{2, \alpha}$-norms of $\Phi_{+}$(resp. $\left.\Phi_{+}^{-1}\right)$ on any subdomain of $\overline{D_{+}}($resp. of $[-1,1] \times[0, q])$ away from the corners are bounded by the $C^{2, \alpha}$-norm of $\gamma_{0}$ and the distance of the subdomain to the corners.

Proof. By the generalized Schwarz-Christoffel formula (Pommmerenke $[\mathrm{Po}]$ ), there is a conformal diffeomorphism $f$ which maps the unit disk $\mathbb{D}$ to $D_{+}$. Let $z_{i}$ be such that $f\left(z_{i}\right)=v_{i}, i=1,2,3,4$. It is well-known that $f$ extends as a conformal diffeomorphism on $\overline{\mathbb{D}} \backslash\left\{z_{1}, \ldots, z_{4}\right\}$. Next, by the Schwarz-Christoffel formula, there exists a conformal diffeomorphism $g$ from $\mathbb{D}$ to the rectangle $C_{+}=$ $(-1,1) \times(0, q)$ where $q$ is a fixed positive number determined by $z_{1}, z_{2}, z_{3}$ and $z_{4}$. Again, $g$ is a conformal diffeomorphism on $\overline{\mathbb{D}} \backslash\left\{z_{1}, \ldots, z_{4}\right\}$. It follows that $\Phi_{+}=g \circ f^{-1}$ is a conformal diffeomorphism satisfying the requirement of the lemma. 
Let $\Phi_{+}=u+i v$. The $v \in C\left(\overline{D_{+}}\right)$and $\Delta v=0$ in $D_{+}$. As $\left.v\right|_{\gamma_{0}}=0$ and $\left.v\right|_{(-R-1, R+1) \times\{2\}}=q$. By elliptic regularity, $v$ has a $C^{2, \alpha}$-bound depending on the $C^{2, \alpha}$-norm of $\gamma_{0}$ away from the corners. By the Cauchy-Riemann equations $u_{x}=-v_{y}$ and $u_{y}=v_{x}$, we conclude that $\Phi_{+}$has the same regularity as $v$. Similar estimates hold for $\Phi_{+}^{-1}$ as the Jacobian of $\Phi_{+}$never vanishes on $\overline{D_{+}} \backslash\left\{v_{1}, v_{2}, v_{3}, v_{4}\right\}$.

Consider the vertical lines $\left\{l_{\mu}(y): y \in[0, q]\right\}, \mu \in(-1,1)$, which foliate $C_{+}$. The pull-back $\Gamma_{\mu}^{+}=\left(\Phi_{+}^{-1}\right)\left(l_{\mu}\right)$ foliates $\overline{D_{+}} \backslash\{x= \pm(R+1)\}$. Each leaf $\Gamma_{\mu}^{+}$is a smooth curve starting at $(x, 2)=\Phi_{+}^{-1}(\mu, q)$ vertically and ending at $\Phi_{+}^{-1}(\mu, 0)$ vertically. The $C^{2, \alpha}$-norms of $\Gamma_{\mu}^{+}$are uniformly bounded for $\mu \in\left(-1+\varepsilon_{1}, 1-\varepsilon_{1}\right)$ for any fixed $\varepsilon_{1}>0$.

At each point on $\gamma_{0}$, there passes a unique leaf $\Gamma_{\mu}^{+}$. So $\xi_{0}=\left.\frac{d}{d y}\right|_{y=0} \Gamma_{\mu}^{+}$defines a $C^{1, \alpha}$-vector field along $\gamma_{0}$. Now, similar to the situation of $D_{+}$, we have a conformal diffeomorphism $\Phi_{-}$which maps $D_{-}$onto $C_{-}=(-1,1) \times(-r, 0)$ (for some $r>0)$ so that $\Phi_{-}\left(\gamma_{0}\right)=(-1,1) \times\{0\}$. $\Phi_{-}$enjoys the same regularity as $\Phi_{+}$. Under $\Phi_{-}$, its differential evaluating at $\xi_{0},\left(d \Phi_{-}\right)\left(\xi_{0}\right)$, is a $C^{1, \alpha}$-vector field on $(-1,1) \times\{0\}$. In fact, $d \Phi_{-}(\cdot)$ gives a map from the vector field along $\gamma_{0}$ to the vector field on $(-1,1) \times\{0\}$. We could extend $\left(d \Phi_{-}\right)\left(\xi_{0}\right)$ to become the vertical vector field $V$ on $C_{-}$by letting $V=\left(1+\frac{y}{r}\right)\left[\left(d \Phi_{-}\right)\left(\xi_{0}\right)(x, 0)\right]-\frac{y}{r}(0,1)$ where

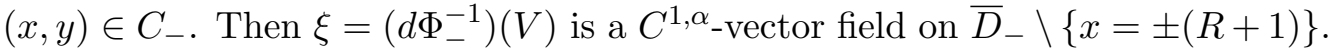
At each point on $\gamma_{0}$ there passes a unique, vertical integral curve $\Gamma_{\mu}^{-}$of $\xi$ which stays inside $D_{-}$and leaves it by hitting a point on $(-R-1, R+1) \times\left\{-\frac{3}{2}\right\}$ vertically. Together with $\Gamma_{\mu}^{+}$we obtain a family of foliations of $\bar{D} \backslash\{x= \pm(R+1)\}$ where each leaf $\Gamma_{\mu}^{+} \cup \Gamma_{\mu}^{-}$is a $C^{1,1}$-curve which is smooth away from $\gamma_{0}$ (because the derivatives of the fields $\xi$ and $\frac{d \Gamma_{\mu}^{+}}{d y}$ may have jump discontinuity along $\gamma_{0}$, $\Gamma_{\mu}^{+} \cup \Gamma_{\mu}^{-}$may not be $C^{2, \alpha}$.)

If we smooth out the discontinuities of $\Gamma_{\mu}^{+} \cup \Gamma_{\mu}^{-}$near $\gamma_{0}$ directly, the obtained smooth curves need not intersect $\gamma_{0}$ transversally. To obtain a smooth foliation satisfying requirement, we fix a tubular neighborhood $\mathcal{N}$ of $\gamma_{0}$, which guarantees that there exists a smooth diffeomorphism $\Phi_{0}$ which maps $\mathcal{N}$ onto the cube $C_{0}=(-1,1) \times(-1,1)$ so that $\Phi_{0}\left(\gamma_{0}\right)=(-1,1) \times\{0\}$. (As the tubular neighborhood is constructed by using the map $(p, t) \mapsto \gamma_{0}(p)+t \nu(p)$, in order the 
diffeomorphism to be smooth we need the smoothness of $\gamma_{0}$. In general, the diffeomorphism is of class $C^{1, \alpha}$ if $\gamma_{0}$ is $C^{2, \alpha}$, and this would be sufficient for our purpose.) Note that the vertical lines $\left\{\tilde{l}_{\mu}(y): y \in[-1,1]\right\}$ with $\mu \in(-1,1)$ foliates $C_{0}$. Then the pull-back $\Gamma_{\mu}^{0}=\left(\Phi_{0}^{-1}\right)\left(\tilde{l}_{\mu}\right)$ foliates $\mathcal{N}$. Each leaf $\Gamma_{\mu}^{0}$ is a smooth curve starting at $\left(\Phi_{0}^{-1}\right)(\mu, 1)$, ending at $\left(\Phi_{0}^{-1}\right)(\mu,-1)$ and intersecting $\gamma_{0}$ vertically. Moreover, for each $\mu \in(-1,1)$, there exists a unique $\mu_{+}$such that $\left(\Phi_{0}^{-1}\right)(\mu, 1)$ is one point of $\Gamma_{\mu_{+}}^{+}$and a unique $\mu_{-}$such that $\left(\Phi_{0}^{-1}\right)(\mu,-1)$ is one point of $\Gamma_{\mu_{-}}^{-}$. Thus, for each $\mu \in(-1,1)$, we obtain a curve $\Gamma_{\mu}$ by linking $\Gamma_{\mu_{+}}^{+}$and $\Gamma_{\mu}^{0}$ at the point $\left(\Phi_{0}^{-1}\right)(\mu, 1)$, and linking $\Gamma_{\mu_{-}}^{-}$and $\Gamma_{\mu}^{0}$ at the point $\left(\Phi_{0}^{-1}\right)(\mu,-1)$. After smoothing out the corners at $\left(\Phi_{0}^{-1}\right)(\mu, 1)$ and $\left(\Phi_{0}^{-1}\right)(\mu,-1)$, we obtain a smooth foliation of $\bar{D} \cap\{-R-1+\delta \leq x \leq R+1-\delta\}$ (for any fixed small $\delta>0$ ) so that each leaf $\Gamma_{\mu}(y), y \in[-r, q]$ (using the same notation) with $\mu$ in some subinterval of $(-1,1)$ intersects $\gamma_{0}$ transversally. The $C^{2, \alpha}$-norms of the leaves are uniformly bounded and the transversal angles are uniformly bounded away from zero on $\bar{D} \cap\{-R-1+\delta \leq x \leq R+1-\delta\}$.

It remains to fulfill (3). We observe that for any point $x$ on the upper boundary there passes a unique $\Gamma_{\mu}$ which ends up at a point in $[-R-1+\delta, R+1-\delta] \times\left\{-\frac{3}{2}\right\}$ whose $x$-coordinate equals to, say, $\phi(x)$. The map $\phi$ is a homeomorphism on $[-R-1+\delta, R+1-\delta]$. We can extend our foliation on $\bar{D} \cap\{-R-1+\delta \leq x \leq$ $R+1-\delta\}$ to $[-R-1+\delta, R+1-\delta] \times[-2,2]$ by prolonging each $\Gamma_{\mu}$ by the line segment connecting $\left(\phi(x),-\frac{3}{2}\right)$ and $(x,-2)$. After smoothing out the corners at $\left(\phi(x),-\frac{3}{2}\right)$ we obtain, finally, a foliation on $[-R-1+\delta, R+1-\delta] \times[-2,2]$ whose leaves are still denoted by $\Gamma_{\mu}(y), y \in[-r-1, q]$. We may scale $[-r-1, q]$ to $[-1,1]$ and fix a small number $\varepsilon_{2}>0$ such that the foliation $\mathcal{F}$ consisting of all $\Gamma_{\mu}, \mu \in\left[\mu_{1}, \mu_{2}\right]$, where $\mu_{1}=-1+\varepsilon_{2}, \mu_{2}=1-\varepsilon_{2}$, covers $\left[-R-\frac{1}{2}, R+\frac{1}{2}\right] \times[-2,2]$. All $\Gamma_{\mu}$ are uniformly bounded in $C^{2, \alpha}$-norm. This foliation satisfies (1)-(4) of our requirement.

According to Huisken $[\mathrm{H}]$, the (CSP) for fixed end points has global solutions which evolves to the line segment connecting the endpoints. So for each $\Gamma_{\mu}$ has a global solution $\Gamma_{\mu}(t)$ which evolves to the vertical line segment $\{(x, y): y \in$ $[-2,2]\}$, where $x$ satisfies $\Phi_{+}(x, 2)=(\mu, q)$. Given any large $K>0$, for each $\Gamma_{\mu}$ there exists some time $T_{\mu}$ such that the slope of $\Gamma_{\mu}(t)$ has absolute value greater than $K$. As $\mu \mapsto \Gamma_{\mu}$ is continuous from $\left[\mu_{1}, \mu_{2}\right]$ to $C^{2, \alpha}([-1,1])$, a compactness 
argument shows that there is a uniform time $T$ such that all slopes of $\Gamma_{\mu}(t)$ have absolute value greater than $K$, for all $\mu \in\left[\mu_{1}, \mu_{2}\right]$ and $t \geq T$.

To show that $\gamma_{t}$ evolves into a graph over $(-R, R)$ at $t=T$ we use an argument from Angenent [A2]. We rotate the foliation $\mathcal{F}$ counterclockwise by a small angle so that the new leaves are still transversal to $\gamma_{0}$ in $\left(-R-\frac{1}{2}, R+\frac{1}{2}\right) \times[-2,2]$. Denote this foliation by $\mathcal{F}^{-}$. Each leaf of $\mathcal{F}^{-}$will evolve to a line segment with negative slope $m_{-}$. Similarly, we obtain a smooth foliation $\mathcal{F}^{+}$by rotating $\mathcal{F}$ clockwise. The slopes of the leaves of $\mathcal{F}^{+}$converge to a positive $m_{+}$. Then the initial $\gamma_{0}$ is pinched between $\Gamma_{\mu}^{+}$and $\Gamma_{\mu}^{-}$at each point (see Figure 2).
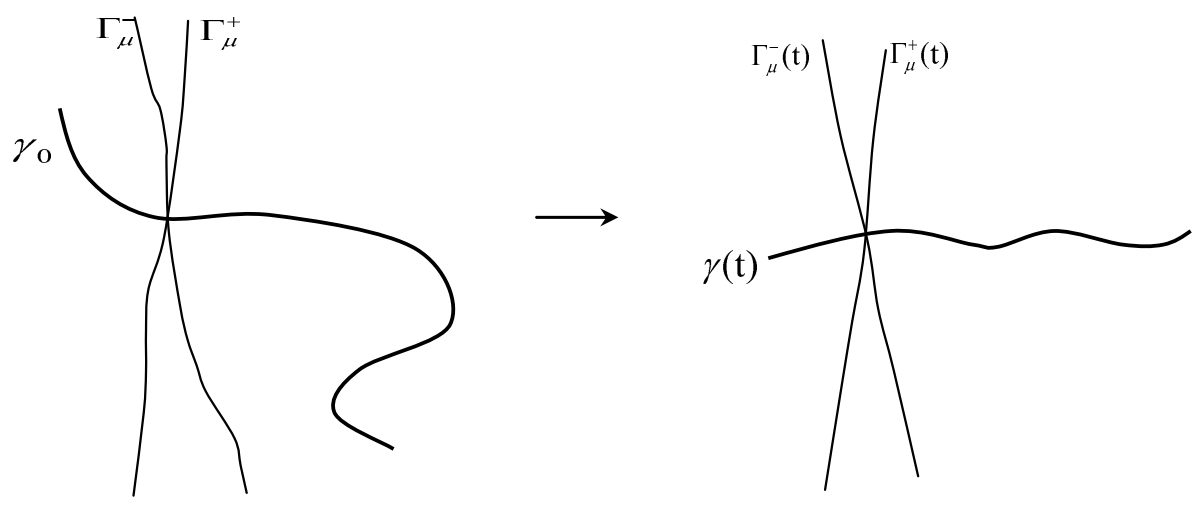

Figure 2. foliations.

Now, let $\gamma_{0}$ and $\mathcal{F}^{ \pm}$evolve with time. As over $\left(-R-\frac{1}{2},-R\right)$ and $\left(R, R+\frac{1}{2}\right), \gamma_{0}$ is always a graph and its intersection points with $\Gamma_{\mu}^{+}\left(\Gamma_{\mu}^{-}\right.$resp.) are exactly once and transversal, by the Sturm oscillation theorem $\gamma_{t}$ intersects $\Gamma_{\mu}^{+}\left(\Gamma_{\mu}^{-}\right.$resp.) exactly once transversally at any later instance. At $t=T$ the slope of $\Gamma_{\mu}^{+}$is uniformly close to $m_{+}$and the slope of $\Gamma_{\mu}^{-}$is uniformly close to $m_{-}$. As $\gamma_{t}$ is pinched between these two curves, its slope cannot become vertical. So it is a graph over $[-R, R]$, see Figure 2 .

Proof of Theorem 1. Applying the above arguments to our solution of (CSP) on each cell $\left(a_{n}, b_{n}\right) \times(-2,2), n \in \mathbb{N}^{*}$, we find a uniform time $T$ such that $\gamma_{T}$ becomes a graph $\left(x, u^{*}(x)\right)$. By the conclusion in Section 1, (1) holds for 
$u_{0}=u^{*}$, that is,

$$
\lim _{R \rightarrow \infty} \sup _{x_{0}} \frac{1}{2 R}\left|\int_{x_{0}-R}^{x_{0}+R} u^{*}(x) d x\right|=0 .
$$

Then Theorem 1 follows immediately from Nara-Taniguchi's result in [NT2].

\section{Discussion}

We note that some conditions in our main theorem look very artificial. A natural question is, how about the long time behavior of $\gamma_{t}$ if $l=\infty$ ? Since there is no explicit estimate for the time a general bounded curve takes to evolve into a graph, we cannot obtain a uniform lower bound of the time for the curves $\mathcal{C}_{i}(1 \leq i \leq \infty)$ to flow into a graph and hence cannot show that $\gamma_{t}$ evolves into an entire graph at finite time. As a partial answer to the above question, we give an example showing that the regularity of the initial curve alone is not enough to ensure the curve becomes an entire graph under the (CSP). Additional information such as the geometry of the regions $D_{ \pm}$also plays a role.

Consider the following curve in Figure 3, which is formed by connecting the negative $x$-axis and a sequence of length-varying cells of the form (see Figure 4) where the rectangles

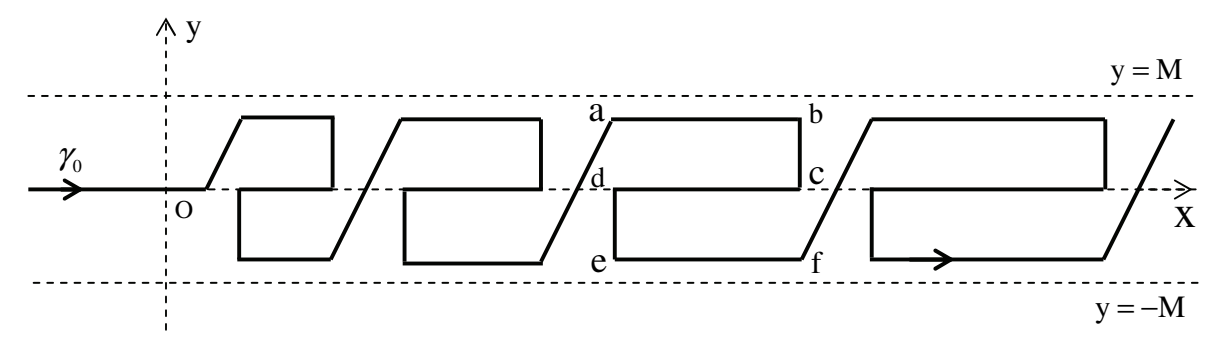

Figure 3. An example.

$a b c d$ and $c d e f$ are congruent. As $p$ increases, the length $L=\overline{a b}$ of a cell increases by 1 unit each time as the cell prolongs along the $x$-axis. Clearly, (2) is satisfied, and by suitably smoothing out the corners, all $C^{k}$-norms of $\gamma_{0}$ can be made to be uniformly bounded. The (CSP) starting at the $\gamma_{0}$ will not evolve into an entire graph in finite time. To see this, consider an ellipse inside the rectangle abcd with area $\pi L$. By the curve shortening flow, the area decreases at rate $2 \pi$, [CZ2]. So 


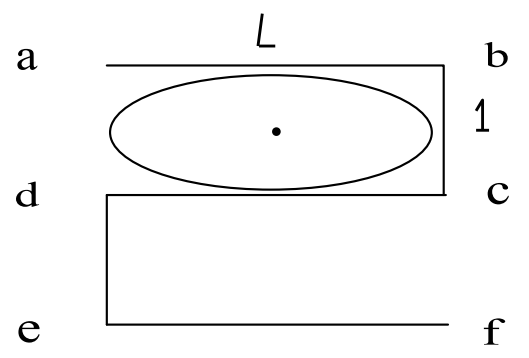

Figure 4. A cell.

the ellipse would shrink to a point at $T=\pi L /(2 \pi)=L / 2$. This means that the $L$-th cell of $\gamma_{0}$ cannot become a graph in $[0, L / 2]$.

\section{REFERENCES}

[AW] S.J. Altschuler \& L.F. Wu, Convergence to translating solutions for a class of quasilinear parabolic boundary problems, Math. Ann. 295 (1993), 761-765.

[A1] S. Angenent, The zero set of a solution of a parabolic equation, J. Reine Angew. Math. 390 (1988), 79-96.

[A2] S. Angenent, Parabolic equations for curves on surfaces II. Intersections, blow up and generalized solutions, Ann. of Math. 133 (1991) 171-215.

[CZ1] K.S. Chou \& X.P. Zhu, Shortening complete plane curves. J. Differential Geom. 50 (1998), no. $3,471-504$.

[CZ2] K.S. Chou \& X.P. Zhu, The curve shortening problem. Chapman \& Hall/CRC, Boca Raton, FL, 2001.

[EH1] K. Ecker \& G. Huisken, Mean curvature evolution of entire graphs, Ann. of Math. 130 (1989) 453-471.

[EH2] K. Ecker \& G. Huisken, Interior estimates for hypersurfaces moving by mean curvature, Invent. Math. 105 (1991) 547-569.

[GH] M. Gage \& R. Hamilton, The heat equation shrinking convex plane curves, J. Differential Geom. 23 (1986) 69-96.

[G] M. A. Grayson, The heat equation shrinks embedded curves to round points, J. Differential Geom. 26 (1987) 285-314.

[H] G. Huisken, A distance comparison principle for evolving curves, Asian J. Math. 2(1998) 127-134.

[HS] N. Hungerbühler \& K. Smoczyk, Soliton solutions for the mean curvature flow, Differential Integral Equations 13(2000), 1321-1345.

[I] N. Ishimura, Curvature evolution of plane curves with prescribed opening angle, Bull. Austral. Math. Soc. 52(1995) 287-296. 
[NT1] M. Nara \& M. Taniguchi, Stability of a traveling wave in curvature flows for spatially nondecaying perturbations, Discrete Contin. Dyn. Syst. 14 (2006) 203-220.

[NT2] M. Nara \& M. Taniguchi, The condition on the stability of stationary lines in a curvature flow in the whole plane, J. Differential Equations 237(2007) 61-76.

[P] A. Polden, Evolving curves, Honours thesis, Australian National University, 1991.

[Po] C. Pommerenke, Boundary behaviour of conformal maps, Grundlehren der Mathematischen Wissenschaften 299, Springer-Verlag, Berlin, 1991.

[S] G. Sapiro, Geometric partial differential equations and image analysis, Cambridge University Press, Cambridge, 2001.

[St] N. Stavrou, Selfsimilar solutions to the mean curvature flow, J. Reine Angew. Math. 499(1998) 189-198.

[V] A. Visintin, Models of phase transitions, Progress in Nonlinear Partial Differential Equations and Their Applications 28, Birkhäuser, Boston, 1996.

\title{
Xiao-Liu Wang \\ Department of Mathematics \\ Southeast University \\ Nanjing, P.R.C., 210096. \\ E-mail: xlwang.seu@gmail.com
}

\author{
Wei-Feng Wo \\ Department of Mathematics \\ Ningbo University \\ Ningbo, P.R.C., 315211. \\ E-mail: woweifeng@nbu.edu.cn
}

